\title{
Circumcision status at HIV infection is not associated with plasma viral load in men: analysis of specimens from a randomized controlled trial
}

Stephanie M. Davis ${ }^{1,5^{*}}$ D, Sherri Pals ${ }^{1}$, Chunfu Yang ${ }^{1}$, Elijah Odoyo-June ${ }^{2}$, Joy Chang ${ }^{1}$, Maroya Spalding Walters ${ }^{1}$, Walter Jaoko ${ }^{3}$, Naomi Bock' ${ }^{1}$ Larry Westerman ${ }^{1}$, Carlos Toledo ${ }^{1}$ and Robert C. Bailey ${ }^{4}$

\begin{abstract}
Background: Male circumcision provides men with approximately $60 \%$ protection from acquiring HIV infection via heterosexual sex, and has become a key component of HIV prevention efforts in sub-Saharan Africa. Possible mechanisms for this protection include removal of the inflammatory anaerobic sub-preputial environment and the high concentration of Langerhans cells on the inside of the foreskin, both believed to promote local vulnerability to HIV infection. In people who do acquire HIV, viral load is partially determined by infecting partner viral load, potentially mediated by size of infecting inoculum. By removing a portal for virion entry, prior male circumcision could decrease infecting inoculum and thus viral load in men who become HIV-infected, conferring the known associated benefits of slower progression to disease and decreased infectiousness.

Methods: We performed an as-treated analysis of plasma samples collected under a randomized controlled trial of male circumcision for HIV prevention, comparing men based on their circumcision status at the time of HIV acquisition, to determine whether circumcision is associated with lower viral load. Eligible men were seroconverters who had at least one plasma sample available drawn at least 6 months after infection, reported no potential exposures other than vaginal sex and, for those who were circumcised, were infected more than 6 weeks after circumcision, to eliminate the open wound as a confounder. Initial viral load testing indicated that quality of pre-2007 samples might have been compromised during storage and they were excluded, as were those with undetectable or unquantifiable results. Log viral loads were compared between groups using univariable and multivariable linear regression, adjusting for sample age and sexually transmitted infection diagnosis with 3.5 months of seroconversion, with a random effect for intraindividual clustering for samples from the same man. A per-protocol analysis was also performed.
\end{abstract}

Results: There were no viral load differences between men who were circumcised and uncircumcised at the time of HIV infection (means 4.00 and $4.03 \log _{10}$ copies/mL respectively, $p=.88$ ) in any analysis.

Conclusion: Circumcision status at the time of HIV infection does not affect viral load in men.

Trial registration: The original RCT which provided the samples was ClinicalTrials.gov trial NCT00059371.

Keywords: Circumcision, HIV prevention, Viral load, Clinical trials, Intervention

\footnotetext{
* Correspondence: smdavis@cdc.gov

'Division of Global HIV/AIDS and Tuberculosis, US Centers for Disease

Control, Atlanta, GA, USA

${ }^{5}$ Division of Global HIV and TB, HIV Prevention Branch US Centers for Disease

Control and Prevention, 1600 Clifton Rd. NE Mail Stop E-04, Atlanta, GA

30329, USA

Full list of author information is available at the end of the article
}

(c) The Author(s). 2018 Open Access This article is distributed under the terms of the Creative Commons Attribution 4.0 International License (http://creativecommons.org/licenses/by/4.0/), which permits unrestricted use, distribution, and

reproduction in any medium, provided you give appropriate credit to the original author(s) and the source, provide a link to the Creative Commons license, and indicate if changes were made. The Creative Commons Public Domain Dedication waiver (http://creativecommons.org/publicdomain/zero/1.0/) applies to the data made available in this article, unless otherwise stated. 


\section{Background}

Male circumcision (MC) has been shown in randomized controlled trials (RCTs) to confer approximately 60\% protection to men from acquiring HIV infection via heterosexual sex [1-3]. It has become a key component of HIV prevention efforts in sub-Saharan Africa, with nearly 15 million voluntary medical male circumcisions (VMMC) performed since 2008 [4]. Widely-accepted possible mechanisms for the protective effect include: the removal of the inner foreskin's high concentration of Langerhans cells, through which HIV can access the immune system; the foreskin's vulnerability to microabrasions; the elimination of the anaerobic sub-preputial environment that supports a pro-inflammatory microbiome; and the greater incidence of genital ulcer disease (GUD) among uncircumcised men [5].

Nevertheless, many circumcised men will ultimately become HIV-infected. Determinants of plasma viral load (VL) set point among infected individuals are not entirely understood, but in addition to well-established associations like host immunogenetics [6] and viral genotype [7], it is associated with VL in "source" partners [8-10]. Although this relationship is sometimes attributed to viral genotype also, another suggested mechanism is that high source partner VL results in a higher infecting inoculum, which then results in higher VL in the newly-infected partner [11, 12]. (The majority of HIV infections resulting from heterosexual sex are believed to result from a single virion $[9,13]$, so the effect of inoculum size on VL is not hypothesized to result directly from a higher initial virion population. Instead, a larger inoculum may lead to increased host T-cell activation, which in turn enhances replication of the ultimately 'successful' virion [10]). Supporting evidence for inoculum size as an independent determinant of VL includes the association between source partner genital tract factors which would affect magnitude of viral shedding, such as bacterial vaginosis and genital herpes suppression, and VLs in seroconverting partners [12].

If source inoculum affects $\mathrm{VL}$, the same mechanisms that confer partial protection against infection could operate in circumcised men who do become infected to decrease the proportion of virions breaching their skin, lowering inoculum and thus VL. Fig. 1 illustrates this proposed causal chain. If present, such an effect could confer substantial population-level benefit, as VL is a major determinant of both progression to clinical disease and infectiousness [14].

Evidence for the plausibility of this result comes from one study that found MC in genotype-confirmed male source partners was associated with lower HIV VLs among the female partners they infected $[10,15]$ (adjusted mean difference $=-.63 \log _{10}$ copies $/ \mathrm{mL}, p=.03$ ). The authors hypothesized the above mechanism in reverse: lower innocula transferred to female partners due to the removal of the male's infectious foreskin Langerhans cells via MC. This study did not find evidence of lower VL set points associated with MC among men infected by female source partners, but this outcome was limited by small sample size $(N=55)$ and the fact that participants were not randomized with respect to circumcision status.

We used stored plasma samples from the original RCT of MC for HIV prevention conducted in Kisumu, Kenya [2], to investigate whether MC status of male participants who became HIV-infected was associated with their VL.

\section{Methods}

Enrollment, randomization, sample collection and follow-up in the Kisumu-based RCT

Detailed methods of the RCT are described elsewhere [2] (ClinicalTrials.gov, NCT00059371). Briefly, it was conducted in Kisumu district, western Kenya. Male participants were recruited via newspapers, radio, fliers and street shows and enrolled between 2002 and 2005. Inclusion criteria included being an uncircumcised, HIV-negative, sexually active, 18-24-year-old Kisumu resident. Exclusion criteria included contraindications to, and absolute indications for, surgical MC. An opaque envelope system was used to produce 1:1 allocation between arms. Plasma HIV testing was performed and self-reported HIV exposure data collected at 1, 3, and 6 months after randomization, then at 6-month intervals. The trial was stopped early in December 2006 due to efficacy. Free MC was then offered to all interested

\begin{tabular}{|c|c|c|c|c|c|c|}
\hline $\begin{array}{l}\text { Number of } \\
\text { portals of } \\
\text { entry reduced } \\
\text { by removal of } \\
\text { foreskin }\end{array}$ & $\Rightarrow$ & $\begin{array}{l}\text { Decreased } \\
\text { total HIV } \\
\text { inoculum } \\
\text { passing skin } \\
\text { barrier }\end{array}$ & $\begin{array}{l}\text { Decreased } \\
\text { responsive } \\
\text { T-cell } \\
\text { activation }\end{array}$ & $\Rightarrow$ & $\begin{array}{l}\text { Decreased early } \\
\text { replication of the } \\
\text { 'successful' single } \\
\text { virion which } \\
\text { establishes } \\
\text { infection }\end{array}$ & $\begin{array}{l}\text { Decreased } \\
\text { viral load }\end{array}$ \\
\hline
\end{tabular}

Fig. 1 Potential relationship between circumcision status at HIV infection and viral load 
participants throughout the follow-up period, causing crossover from the control group. Semi-annual follow-up visits continued through September 2010. The dataset is publically available [16].

\section{HIV testing and determination of seroconversion and infection dates}

Two rapid tests were used at each visit, Determine HIV 1/2 (Abbott Diagnostic Division, Hoofddorp, Netherlands) and Unigold Recombigen (Trinity Biotech, Wicklow, Ireland). If one or both was positive, serum was sent for double enzyme-linked immunosorbent assay testing (Detect HIV 1/2, Adaltis Inc., Montreal, Canada and Recombigen HIV 1/2, Trinity Biotech, Wicklow, Ireland). If at least one ELISA was positive, final confirmatory testing was done by line immunoassay (INNO-LIA HIV 1/2, Immunogenetics NV, Ghent, Belgium). For participants confirmed as HIV positive, the first visit with at least one positive rapid test was designated the HIV seroconversion visit. We calculated the presumed infection date as the date midway between the final negative test and the HIV seroconversion visit.

\section{Other data}

Additional data collected at enrollment and in follow-up included MC procedure date, behavioral and other HIV risk factors, and sexually transmitted infection (STI) diagnoses. High-risk sexual behavior within the 6 months before a visit was defined as self-report of $>2$ female sex partners, exchanging gifts or money for sex, or using a condom "less than half the time".

STI testing included serum rapid plasma reagin (RPR) (Becton Dickinson) with T. pallidum particle agglutination (TPHA) confirmation for syphilis and HSV-2 serology; and urine polymerase chain reaction (PCR) for $N$. gonorrhoeae and C. trachomatis. Men with urethral discharge also had urethral swabs for $N$. gonorrhoeae culture and PCR, C. trachomatis PCR (Roche Diagnostics), and T. vaginalis culture (In pouch test). Any genital ulcers were swabbed for $H$. ducreyi culture, and PCR and HSV-2 PCR testing using the Roche Multiplex PCR system. Men were defined as having an STI if any tests were positive except a positive RPR with negative TPHA. The study did not collect data on antiretroviral therapy (ART) initiation among participants; between 2005 and 2011, the Kenyan National HIV Treatment guidelines set a CD4 threshold for ART of $<=200 / \mathrm{mm}^{3}$.

\section{Inclusion and exclusion criteria for HIV VL analysis}

Individual inclusion criteria for this analysis were: seroconversion during the trial or follow-up; and having at least one available plasma sample drawn at least 6 months after infection date and, for those who became circumcised after infection, drawn 6 months after MC (to eliminate bias from the procedure causing transient VL increases). Individual exclusion criteria were: potential HIV exposures other than vaginal sex at any time (sex with men, intravenous drug use, or blood transfusion); a still-unhealed $\mathrm{MC}$ wound less than 3 weeks prior to infection; and for circumcised men, infection date fewer than 6 weeks after MC (unhealed wounds may have facilitated infection among men who resumed sex earlier).

Sample exclusion criteria were also applied. Samples drawn before Jan 1, 2007 were excluded from the final analysis because all but five (of 85) had undetectable or unquantifiable VLs, correlations described below between sample age and VL suggested older samples were subject to degradation, and none of these samples had second aliquots available for quality control. Finally, samples with undetectable or unquantifiable results were also ultimately excluded (see Data Analysis section for methods and reasoning). Among eligible men, the first eligible samples, up to three, were used.

\section{Sample size}

The anticipated sample size based on total available aliquots was 123 men (82 circumcised and 41 uncircumcised), which provided $80 \%$ power to detect a between-group difference in mean VL of .54 standard deviations, equivalent to $.93 \log _{10}$ copies/mL [12]. Power calculations were done using SAS 9.3 PROC POWER TWOSAMPLEMEANS with a two-sample t-test.

\section{Sample storage and testing}

Plasma samples were not tested at the time of collection, and were not thawed at any time before testing. They were stored at $-80{ }^{\circ} \mathrm{C}$, initially at the Kenya Medical Research Institute in Kisumu, and permanently at the Chicago Developmental Center for AIDS Research. VL testing was performed by the International Laboratory Branch of the US Centers for Disease Control's Division of Global HIV and Tuberculosis in Atlanta, Georgia, using the Abbott HIV-1 Real Time HIV-1 assay on the Abbott m2000 instrument, with a lower limit of detection of 150 copies $/ \mathrm{mL}$ with a $200 \mu \mathrm{L}$ plasma input volume. All samples with an available second aliquot were retested and results compared against initial values, with a difference threshold of $\leq 0.5 \log _{10}$ copies $/ \mathrm{mL}$ for concurrence.

\section{Data analysis}

In the primary (as-treated) analysis, men were classified as circumcised or uncircumcised based on whether they had been circumcised through the RCT's services before infection. The secondary (per-protocol) analysis restricted the comparison to men remaining in their original randomization group. Analysis was performed 
using SAS 9.3 on $\log _{10}$-transformed VL results from the first round of testing.

Once a high proportion of samples with undetectable or unquantifiable (VL detected at a concentration below the quantifiability threshold, $2.18 \log$ copies $/ \mathrm{mL}$ ) results was identified, methods for handling these values were devised. Prior to the second round of testing, it was pre-specified that all such samples would be retested if a second aliquot was available, and the values would be included in the analysis if at least $80 \%$ of their retested samples were again either undetectable or unquantifiable, increasing confidence that these represented true plasma values rather than degradation of the aliquots.

To compare characteristics between groups, we used Fisher's exact Chi-square test for categorical variables, and $\mathrm{t}$-tests for continuous variables. Pearson correlation coefficients were used to examine the relationship between sample age and log VL.

PROC MIXED was used to test for differences in log VL between groups, with a random effect for individual to account for the correlation between samples from the same man, This approach yields results similar to an analysis on log VL means computed for each man and then compared between groups, but also allows for the use of sample-level covariates such as sample age.

\section{Results}

Specimen collection dates for included samples ranged from January 10, 2007 to September 30, 2010. VL results were concurrent between aliquots for all retested quantifiable samples, but samples with initially undetectable or unquantifiable VL results did not meet the prespecified criteria and were excluded, leaving 124 included samples representing 63 seroconverting participants (37 circumcised and 26 uncircumcised). Twenty-three men had only one sample. With the reduction in size, the analyzed sample had $80 \%$ power to detect a between-group difference of .73 standard deviations, or $1.26 \log _{10}$ copies $/ \mathrm{mL}$. Log viral load results had a range of 2.33 to 6.21, with a median of 4.04 (IQR 3.57-4.53). For men with two or three results, the intraclass correlation between log viral loads was strong at 0.63 . Table 1 compares key demographic and risk characteristics, and sample-level characteristics of draw timing and age at testing, for circumcised and uncircumcised participants. Groups were similar demographically. Circumcised men had a nonsignificant higher prevalence of risky behavior and lower prevalence of STI. Samples were similar in draw timing and age at testing.

A strong linear correlation was found between $\mathrm{VL}$ and sample age (Fig. $2 ; r=-.48, p<.0001, \mathrm{R}^{2}=.23$ ); a quadratic term was tested for significance to assess for a nonlinear association, but was not significant and was therefore dropped. No significant correlation was found between VL and duration of infection at time of blood draw (Fig. 3; $r=.07, p=0.40$ ).

No significant difference in $\log$ VL was found between circumcised and uncircumcised men in the as-treated (means 4.00 and $4.03 \log$ copies/mL respectively, $p=.88$ ) or per-protocol (means 4.06 and $3.99 \log$ copies $/ \mathrm{mL}$, $p=.76$ ) analyses. This remained true after adjusting for STI diagnosis within 3.5 months of seroconversion and age of sample, for both the as-treated (means 3.96 and $4.09 \log$ copies $/ \mathrm{mL}, p=.47$ ) and the per-protocol (means 3.99 and $4.04 \log$ copies $/ \mathrm{mL}, p=.80$ ) analysis.

\section{Discussion}

Our results do not support an effect of circumcision status larger than $1.26 \log _{10}$ copies $/ \mathrm{mL}$ at the time of seroconversion on VL after 6 months. This does not rule out a clinically significant effect: a 1 log difference in VL was found in Uganda to be associated with an adjusted

Table 1 Characteristics of circumcised and uncircumcised HIV-1 seroconverters and their samples (as-treated analysis)

\begin{tabular}{|c|c|c|c|}
\hline Characteristic & Circumcised mean (SD) or \% (n) & Uncircumcised mean (SD) or \% (n) & $p$-value ${ }^{a}$ \\
\hline \multicolumn{4}{|l|}{ Individual-level ( $N=37$ circumcised, 26 uncircumcised) } \\
\hline Mean age (years) & $20.0(1.5)$ & $20.4(1.6)$ & .32 \\
\hline Mean educational attainment (years) & $9.3(2.1)$ & $10.0(2.2)$ & .20 \\
\hline STI diagnosis within 3.5 months of seroconversion - \% (n) & $13.5(5)$ & $34.6(9)$ & .08 \\
\hline High-risk sex within 6 months of seroconversion - \% (n) & $70.3(26)$ & $53.8(14)$ & .20 \\
\hline \multicolumn{4}{|l|}{ Sample-level ( $N=72$ circumcised, 52 uncircumcised) } \\
\hline Mean (days) between seroconversion and draw date & $516.2(210.7)$ & $558.8(330.3)$ & .42 \\
\hline Percent $(\mathrm{n})$ drawn $<12$ mo. post seroconversion & $26.4(19)$ & $34.6(18)$ & .28 \\
\hline Percent (n) drawn 12-17 mo. post seroconversion & $31.9(23)$ & $23.1(12)$ & \\
\hline Percent (n) drawn > 18 mo. post seroconversion & $27.8(20)$ & $19.2(10)$ & \\
\hline Percent (n) drawn > 24 mo. post seroconversion & $13.9(10)$ & $23.1(12)$ & \\
\hline Mean age (days) of sample(s) at testing & $2646.5(367.5)$ & $2702.4(375.5)$ & .41 \\
\hline
\end{tabular}

${ }^{a}$ Tests for continuous variables were done using t-tests; tests for categorical variables were done using exact Chi-square tests 


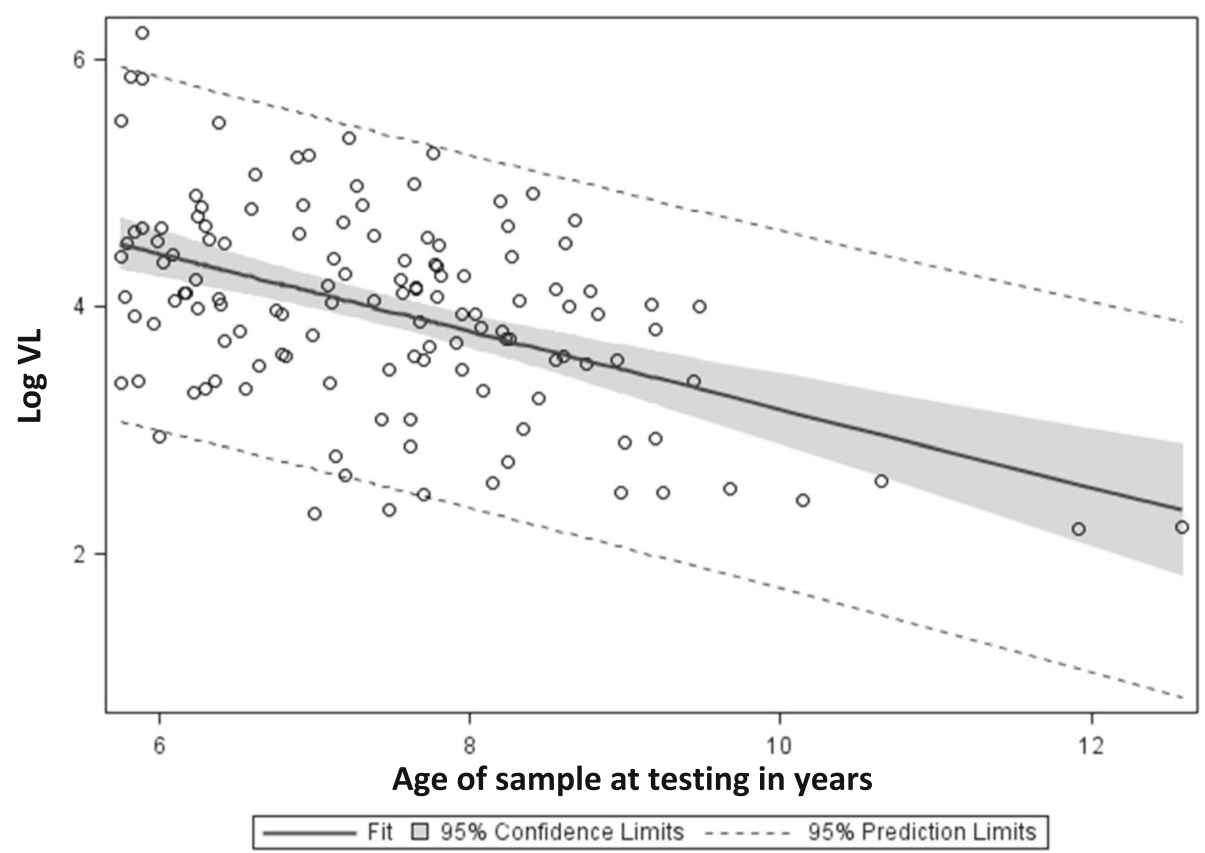

Fig. 2 Relationship between age of sample and $\log _{10}$ viral load

hazard ratio of 3.09 for death and 2.75 for progression to AIDS [17] and the $1 \mathrm{log}$ difference between a VL of 10,000 and 100,000 copies/mL has been modeled to be associated with approximately a 5-year difference in asymptomatic survival [18]. However, this finding is consistent with the observational results reported by
Lingappa et al. in participants from southern and eastern African countries, and represents gold-standard data due to randomization which is unlikely to become available from future studies. It also helps establish the assumptions needed for sample size planning for any observational studies further examining the issue. Given the

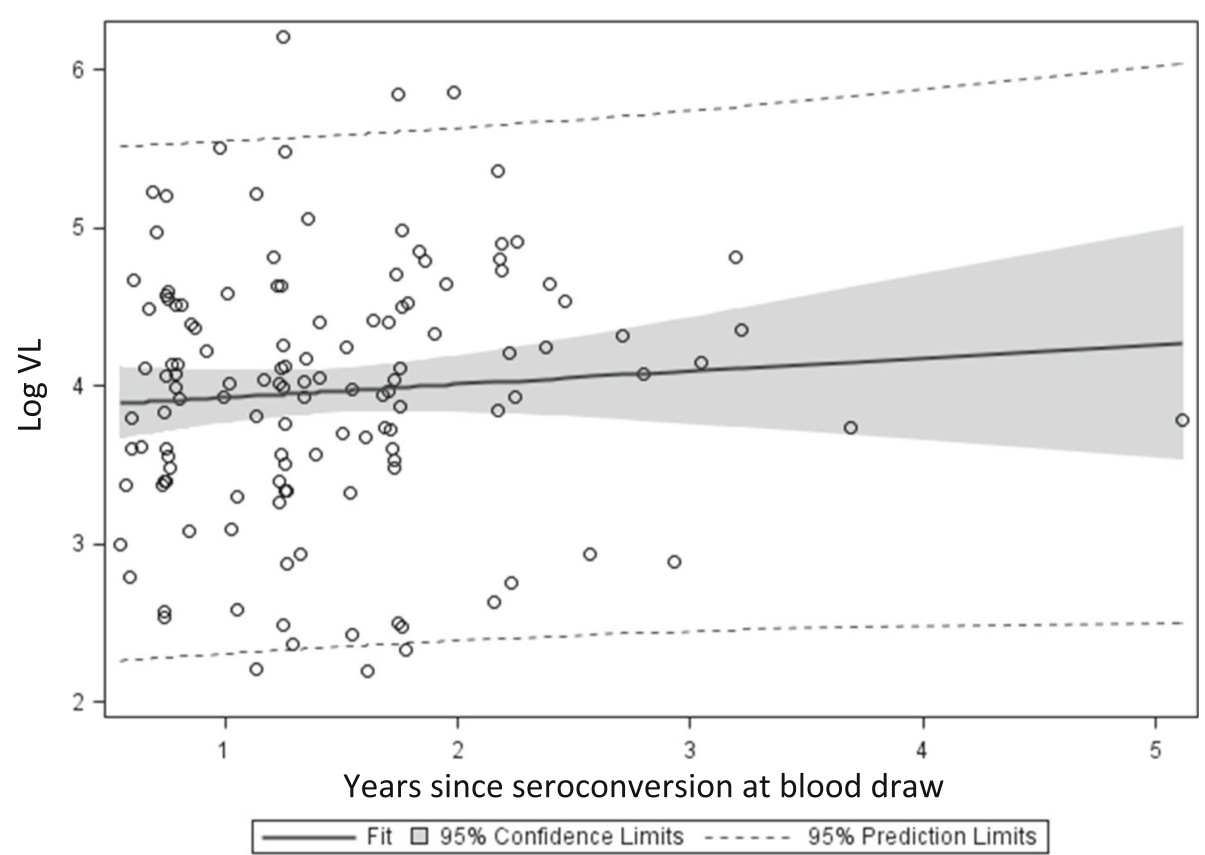

Fig. 3 Relationship between time since seroconversion and $\log _{10}$ viral load 
mechanistic biological nature of the proposed effect, results are also likely to be valid across populations.

If the lack of observed effect reflects biological fact, the explanation may lie in the set of portals of entry available to HIV virions on the male genitalia. In addition to the foreskin, the glans, urethral mucosa and possibly fossa navicularis are all portals across which translocation of cell-associated HIV-1 has been demonstrated in vivo [19]. Given the low per-act probability of female-to-male transmission through any of these portals (4 per 10,000 infectious exposures in a recent systematic review) [20], it seems to follow that the probability of transmission through any single portal is even lower, and thus that in any one act leading to infection, the most likely scenario is that only one portal is involved. Thus circumcision would remove one portal, decreasing infection risk, but would not affect the size of an inoculum passing through a different portal, thus not affecting VL if infectious becomes established. Others have found higher per-act transmission probabilities such as $0.38 \%$ in developing countries in the absence of commercial sex work or genital ulceration, [21] but also at this higher magnitude, a single portal seems likely to be involved in the majority of transmissions.

Conversely, the previously-observed lower VLs in women with circumcised male source partners are more likely to be related to total male viral shedding, which would be expected to come from multiple "portals of exit" during sex, including semen and penile mucosa. Shedding has been shown via coronal lavage to be reduced in HIV-infected men after healing from MC, compared to pre-MC levels [22].

It is also possible that infecting inoculum does not actually impact VL, though this would not explain Lingappa et al.'s [12] observed association between male infecting partner circumcision and lower female partner $\mathrm{VL}$ in their baseline data.

Finally, it is possible that there is a population-level effect of circumcision status at time of infection on men's $\mathrm{VL}$, but that it is mediated by the known protective associations of $\mathrm{MC}$ with risk for genital ulcer disease (GUD) [23], thus decreasing VL indirectly through the same mechanisms postulated for a direct effect. Lingappa et al. [12] did not find an association between HSV-2 serology and VL set point, but thoroughly testing this possibility would require a much larger sample of circumcised and uncircumcised seroconverters with sufficient prevalence of GUD to estimate a true effect.

The major potential confounder is the effect of sample age on VL. Although controlling for this did not impact results, the effect of storage time on VL can have an unpredictable [24] component, which could have caused residual confounding. However, if substantial, this component should have eroded all observed associations; the observed correlations between VL and sample age, and between VLs from the same participant, should not have been seen.

Another potential confounder is ART initiation, which was not captured in the study data. However, between 2005 and 2011, Kenyan National HIV Treatment guidelines set a CD4 threshold for ART of $<=200 / \mathrm{mm}^{3}$ in the absence of clinical indications, and the maximum elapsed time of 5.1 years between calculated infection date and blood draw in our sample makes it unlikely that participants would have progressed to meet this criterion and start treatment. If any did, elimination of samples with undetectable viral load would also have been likely to exclude them. In other respects, although circumcised participants were somewhat more likely to report risky behavior, the difference was not significant, and VLs would not necessarily be higher in infections contracted through high-risk sex.

Finally, for participants with only one sample, stability of VL over time cannot be demonstrated; therefore, VL cannot necessarily be considered equivalent to VL set point.

\section{Conclusions}

Though circumcision in HIV-positive men has an observed association with HIV VL in female partners infected by them, this study provides no evidence that circumcision status at HIV seroconversion affects VL after 6 months in men.

\section{Abbreviations}

ART: Antiretroviral therapy; MC: Male circumcision; RCT: Randomized controlled trial; RPR: Rapid plasma reagin; STI: Sexually transmitted infection; TPHA: T. pallidum particle agglutination; VL: Viral load; VMMC: Voluntary medical male circumcision

Acknowledgements

Not applicable.

\section{Funding}

This research has been supported by the President's Emergency Plan for AIDS Relief (PEPFAR) through CDC. The original RCT of circumcision in Kisumu was funded by the Division of AIDS, NIAD, NIH, and storage of the samples was supported by the Chicago Developmental Center for AIDS Research.

\section{Availability of data and materials}

The dataset supporting the conclusions of this article from the original RCT that provided the samples is publically available at the National Technical Reports Library: https://ntrl.ntis.gov/NTRL/dashboard/searchResults.xhtml?sea rchQuery=Male\%20circumcision. The viral load data generated for this analysis is available from the corresponding author on reasonable request.

\section{Disclaimer}

The findings and conclusions in this paper are those of the authors and do not necessarily represent the views of the US Centers for Disease Control and Prevention or $\mathrm{NIH}$.

\section{Authors' contributions}

All authors have reviewed and approved the final manuscript. SD conceived of the analysis and drafted and revised the manuscript. SP performed all statistical calculations and figure development and revised the manuscript. $C Y$ and LW designed and supervised sample testing and revised the 
manuscript. EO and WJ led local protocol approval and revised the manuscript. MW contributed to analytic design and revised the manuscript. JC performed sample testing. NB and CT revised the manuscript. RB led the original trial, contributed to analytic design, and revised the manuscript.

\section{Ethics approval and consent to participate}

This secondary analysis obtained IRB approval from the Kenyatta National Hospital ethics and research committee, and exemption determinations from CDC Human Subjects Review and a University of Illinois IRB.

\section{Consent for publication}

Not applicable.

\section{Competing interests}

The authors declare that they have no competing interests.

\section{Publisher's Note}

Springer Nature remains neutral with regard to jurisdictional claims in published maps and institutional affiliations.

\section{Author details}

'Division of Global HIV/AIDS and Tuberculosis, US Centers for Disease Control, Atlanta, GA, USA. ²Division of Global HIV/AIDS and Tuberculosis, US Centers for Disease Control, Kisumu, Kenya. ${ }^{3}$ Department of Medical Microbiology, University of Nairobi, Nairobi, Kenya. ${ }^{4}$ Division of Epidemiology and Biostatistics, University of Illinois Chicago School of Public Health, Chicago, IL, USA. ${ }^{5}$ Division of Global HIV and TB, HIV Prevention Branch US Centers for Disease Control and Prevention, 1600 Clifton Rd. NE Mail Stop E-04, Atlanta, GA 30329, USA.

\section{Received: 14 February 2018 Accepted: 17 July 2018}

Published online: 28 July 2018

\section{References}

1. Auvert B, Taljaard D, Lagarde E, Sobngwi-Tambekou J, Sitta R, Puren A. Randomized, controlled intervention trial of male circumcision for reduction of HIV infection risk: the ANRS 1265 Trial. PLoS Med. 2005;2(11):e298.

2. Bailey RC, Moses S, Parker CB, Agot K, Maclean I, Krieger JN. Male circumcision for HIV prevention in young men in Kisumu, Kenya: a randomised controlled trial. Lancet. 2007;369(9562):643-56.

3. Gray RH, Kigozi G, Serwadda D, Makumbi F, Watya S, Nalugoda F. Male circumcision for HIV prevention in men in Rakai, Uganda: a randomised trial. Lancet. 2007;369(9562):657-66.

4. World Health Organization. Voluntary medical male circumcision for HIV prevention in 14 Priority Countries In Eastern And Southern Africa: July 2017 Progress Brief. Accessed online Feb 2, 2017 at http://www.who.int/hiv/pub/ malecircumcision/vmmc-progress-brief-2017/en/.

5. Morris BJ, Wamai RG. Biological basis for the protective effect conferred by male circumcision against HIV infection. Int J STD AIDS. 2012;23(3):153-9. https://doi.org/10.1258/ijsa.2011.011228.

6. Saathoff E, Pritsch M, Geldmacher C, Hoffmann O, Koehler RN, Maboko L, et al. Viral and host factors associated with the HIV-1 viral load set point in adults from Mbeya region. Tanzania J Acquir Immune Defic Syndr. 2010; 54(3):324-30.

7. Hodcroft E, Hadfield JD, Fearnhill E, Phillips A, Dunn D, O'Shea D, et al, on behalf of the UK HIV Drug Resistance Database and the UK CHIC Study. The contribution of viral genotype to plasma viral set-point in HIV infection. PLoS Pathog. 2014 10(5): e1004112. doi: https:/doi.org/10.1371/journal.ppat. 1004112

8. Hollingsworth TD, Laeyendecker O, Shirreff G, Donnelly CA, Serwadda D, et al. HIV-1 transmitting couples have similar viral load set-points in Rakai. Uganda PLoS Pathology. 2010;6(5):e1000876. https://doi.org/10.1371/journal. ppat.1000876.

9. Tang J, Tang S, Lobashevsky E, Zulu I, Aldrovandi G, Allen S, et al. HLA allele sharing and HIV type 1 viremia in seroconverting Zambians with known transmitting partners. AIDS Res Hum Retrovir. 2004;20(1):19-25.

10. Hecht FM, Hartogensis W, Bragg L, Bacchetti P, Atchison R, Grant R, et al. HIV RNA level in early infection is predicted by viral load in the transmission source. AIDS. 2010;24(7):941-5.

11. Shaw GM, Hunter E. HIV transmission. Cold Spring Harb Perspect Med. 2012; 2(11):pii: a006965. https://doi.org/10.1101/cshperspect.a006965.
12. Lingappa JR, Thomas KK, Hughes JP, Baeten JM, Wald A, Farquhar C, et al. On behalf of Partners in Prevention HSV/HIV transmission study team. Partner characteristics predicting HIV-1 set point in sexually acquired HIV-1 among African seroconverters. AIDS Res Hum Retrovir. 2013;29(1):164-71.

13. Keele BF, Giorgi EE, Salazar-Gonzalez JF, Decker JM, Pham KT, Salazar MG, et al. Identification and characterization of transmitted and early founder virus envelopes in primary HIV-1 infection. Proc Natl Acad Sci U S A. 2008; 105(21):7552-7. https://doi.org/10.1073/pnas.0802203105. Epub 2008 May 19

14. Mellors JW, Rinaldo CR Jr, Gupta P, White RM, Todd JA, Kingsley LA. Prognosis in HIV-1 infection predicted by the quantity of virus in plasma. Science. 1996:272:1167-70.

15. Mackelprang RD, Carrington M, Thomas KK, Hughes JP, Baeten JM, Wald A, et al. Host genetic and viral determinants of HIV-1 RNA set point among HIV-1 seroconverters from sub-saharan Africa. J Virol. 2015;89(4):2104-11. https://doi.org/10.1128/JVI.01573-14. Epub 2014 Dec 3

16. Randomized Control Trial of Male Circumcision to Reduce HIV Incidence in Kisumu, Kenya. Accession number PB2012500016. National Technical Reports Library: https://ntrl.ntis.gov/NTRL/dashboard/searchResults. xhtml?searchQuery=Male\%20circumcision

17. Eller MA, Opollo MS, Liu M, Redd AD, Eller LA, Kityo C, et al. HIV type 1 disease progression to AIDS and death in a rural Ugandan cohort is primarily dependent on viral load despite variable subtype and T-cell immune activation levels. J Inf Dis. 2014;211(10):1574-84.

18. Fraser $C$, Hollingsworth D, Chapman R, de Wolf F, Hange WP, et al. Variation in HIV-1 set-point viral load: epidemiological analysis and an evolutionary hypothesis. PNAS. 2007:104(44):17441-6.

19. Ganor Y, Zhou Z, Bodo J, Tudor D, Leibowitch J, Mathez D, et al. The adult penile urethra is a novel entry site for HIV-1 that preferentially targets resident urethral macrophages. Nature. 2013;6:4.

20. Patel P, Borkowf CB, Brooks JT, Lasry A, Lansky A, Mermin J. Estimating peract HIV transmission risk: a systematic review. AIDS. 2014;28(10):1509-19. https://doi.org/10.1097/QAD.0000000000000298.

21. Boily M-C, Baggaley RF, Wang L, Masse B, White RG, Hayes R, et al. Heterosexual risk of HIV-1 infection per sexual act: a systematic review and meta-analysis of observational studies. Lancet Infect Dis. 2009 Feb;9(2):118-29. https://doi.org/10.1016/S1473-3099(09)70021-0.

22. Odoyo-June E, Rogers JH, Jaoko W, Bailey RC. Changes in plasma viral load and penile viral shedding after circumcision among HIV-positive men in Kisumu, Kenya. J Acquir Immune Defic Syndr. 2013;64(5):511-7. https://doi. org/10.1097/QAl.0b013e3182a7ef05.

23. Mehta SD, Green SJ, Maclean I, Hu H, Bailey RC, Gillevet PM, Spear GT. Microbial diversity of genital ulcer disease in men enrolled in a randomized trial of male circumcision in Kisumu, Kenya. PLoS One. 2012;7(7):e38991. https://doi.org/10.1371/journal.pone.0038991.

24. Sebire K, McGavin K, Land S, Middleton T, Birch C. Stability of human immunodeficiency virus RNA in blood specimens as measured by a commercial PCR-based assay. J Clin Microbiol. 1998;36(2):493-8.

Ready to submit your research? Choose BMC and benefit from:

- fast, convenient online submission

- thorough peer review by experienced researchers in your field

- rapid publication on acceptance

- support for research data, including large and complex data types

- gold Open Access which fosters wider collaboration and increased citations

- maximum visibility for your research: over $100 \mathrm{M}$ website views per year

At $\mathrm{BMC}$, research is always in progress.

Learn more biomedcentral.com/submissions 